\title{
Quantitative single shot and spatially resolved plasma wakefield diagnostics
}

\author{
Muhammad Firmansyah Kasim, ${ }^{1}$ James Holloway, ${ }^{1}$ Luke Ceurvorst,${ }^{2}$ Matthew C. Levy, ${ }^{2}$ \\ Naren Ratan, ${ }^{2}$ James Sadler, ${ }^{2}$ Robert Bingham, ${ }^{3,5}$ Philip N. Burrows, ${ }^{1}$ Raoul Trines, ${ }^{3}$ \\ Matthew Wing, ${ }^{4}$ and Peter Norreys ${ }^{2,3}$ \\ ${ }^{1}$ John Adams Institute, Denys Wilkinson Building, Keble Road, Oxford OX1 3RH, United Kingdom \\ ${ }^{2}$ Clarendon Laboratory, Department of Physics, University of Oxford, \\ Parks Road, Oxford OX1 3PU, United Kingdom \\ ${ }^{3}$ STFC Rutherford Appleton Laboratory, Chilton, Didcot OX11 OQX, United Kingdom \\ ${ }^{4}$ Department of Physics and Astronomy, University College London, \\ Gower Street, London WCIE 6BT, United Kingdom \\ ${ }^{5}$ Department of Physics, University of Strathclyde, Glasgow G4 ONG, United Kingdom
}

(Received 5 May 2015; published 6 August 2015)

\begin{abstract}
Diagnosing plasma conditions can give great advantages in optimizing plasma wakefield accelerator experiments. One possible method is that of photon acceleration. By propagating a laser probe pulse through a plasma wakefield and extracting the imposed frequency modulation, one can obtain an image of the density modulation of the wakefield. In order to diagnose the wakefield parameters at a chosen point in the plasma, the probe pulse crosses the plasma at oblique angles relative to the wakefield. In this paper, mathematical expressions relating the frequency modulation of the laser pulse and the wakefield density profile of the plasma for oblique crossing angles are derived. Multidimensional particle-in-cell simulation results presented in this paper confirm that the frequency modulation profiles and the density modulation profiles agree to within $10 \%$. Limitations to the accuracy of the measurement are discussed in this paper. This technique opens new possibilities to quantitatively diagnose the plasma wakefield density at known positions within the plasma column.
\end{abstract}

DOI: 10.1103/PhysRevSTAB.18.081302

PACS numbers: 42.25.Dd, 52.35.Mw, 52.38.Kd, 42.62.Eh

\section{INTRODUCTION}

When a driver is fired into an underdense plasma, it will generate a large amplitude longitudinal wave in the electron density profile, now widely known as a plasma wakefield [1-3]. Due to gradients in the electron density profile, the driver beams induce electric fields up to tens or hundreds of $\mathrm{GV} / \mathrm{m}[4-7]$. This is three orders of magnitude higher than the electric field produced in conventional accelerators.

If the wakefield is driven by a short laser pulse [8,9], a beat wave [10-12], an electron [13-15], or a proton beam $[16,17]$, all of which propagate with speeds near the speed of light in vacuum, the wakefield also propagates with approximately the same speed as the driver. The large accelerating gradient and high propagation speed of the wakefield makes it possible to use plasma as a basis for a particle accelerator [18-20].

Before 2006, the core part of a plasma accelerator, i.e., the wakefield structure itself, had never been imaged in experiments. The first snapshot of the wakefield was taken using the frequency domain holography method [21]. It used two long chirped pulses to copropagate with the

Published by the American Physical Society under the terms of the Creative Commons Attribution 3.0 License. Further distribution of this work must maintain attribution to the author(s) and the published article's title, journal citation, and DOI. wakefield, as demonstrated in Refs. [22-25]. The phase modulation of the pulse was retrieved from a spectrometer. From the phase modulation profile, the image of a plasma wakefield was obtained. This major advance in diagnostic development has since allowed much greater understanding of the underlying physics. However, one limitation is that the probe pulse copropagates with the wakefield, and so there is an averaging effect of the retrieved wakefield profile. Therefore, it is not possible to diagnose the evolution of the wakefield along the propagation direction.

In 2014, the same group demonstrated a new plasma wakefield diagnostic technique which used two long chirped pulses fired at a certain angle relative to the laser pump pulse [25], which is called the frequency domain streak camera. By measuring the phase modulation of the probe pulse, this technique successfully produced the longitudinal evolution of a wakefield in a single shot. It is useful to detect where the bubble in the wakefield is formed. However, the transverse structure of the wakefield is convolved in the probe's phase modulation. Because this technique does not provide the transverse structure information, there is insufficient information in the data produced in this technique to quantify the wakefield density modulation.

In other experiments, images of plasma wakefields were obtained using shadowgraphy technique [26,27]. In these elegant experiments, probe laser pulses were fired across 
the wakefield in the perpendicular direction. The transverse intensity profiles of the probe pulses represented the second derivative of the wakefields' electron density profiles with respect to position. This allowed the crossing point to be chosen along the propagation distance of the driver. However, because the plasma wakefield propagates with a speed near the speed of light in vacuum, the relative longitudinal position of the probe changes while it is still interacting with the wakefield. This makes it quite difficult to obtain quantitative data from this technique.

In this paper, a novel technique to image and diagnose plasma wakefields, using the concept of photon acceleration, is presented. When photons propagate in a plasma wakefield, their frequencies change according to the gradient of the wakefield's density profile relative to the photons' positions. This phenomenon was first predicted by Wilks et al. [28] and further developed in Refs. [29-32]. By measuring the frequency modulation of the photons, one can retrieve the electron density modulation profile in the wakefield.

In a previous study [33], pioneering results of simulated measurements using photon acceleration using a long copropagating probe pulse were presented. As in frequency domain holography, the setup introduces an averaging effect, which was accounted for in the description of its strengths and limitations. In order to avoid the averaging effect, a new study is presented here where the probe pulse is allowed to propagate with an oblique angle relative to the wakefield. This oblique crossing angle makes it possible to obtain the density modulation profile of the wakefield at certain positions and diagnose the evolution of the wakefield along the propagation distance, thereby overcoming one of the limitations of the previous methods. This technique is also complementary to the frequency domain streak camera technique [25] by providing the quantitative information of the electron density modulation in the wakefield.

If the angle is set correctly, the velocity of the probe pulse in the wakefield's longitudinal direction is the same as the wakefield's group velocity and the longitudinal position of the probe pulse will not change relative to the wakefield. Therefore, an inverse Abel transform [34] can be applied, with the assumption that the wakefield has a cylindrical symmetry. However, the correct angle is not always achievable, especially for the case where the wakefield's speed is greater than the probe's speed. For different crossing angles, the probe pulse's relative longitudinal position changes while crossing the wakefield and an ordinary Abel transform cannot be applied.

In this paper, an expression of a modified Abel transform is derived so that it can be applied for more general crossing angles with its inverse transformation. Results of the simulated measurements are presented for various wakefield amplitudes, frequencies of the probe beam, and crossing angles. In the simulations, two electron density profiles of the wakefield were obtained. The first were obtained directly from the electron density profiles in the simulations, and these are called "actual" profiles throughout this paper. The second were calculated from the electric field profiles of the laser pulse in the simulations. These profiles are called "measured" profiles. The "actual" and "measured" terms will be used often in this paper. These two types of profiles are then compared to confirm the agreement between them. Analysis of the limiting conditions, including the diffraction effect and errordependence on the angle of incidence, are also discussed.

This paper is organized as follows. In Sec. II, a derivation of a transformation for cylindrically symmetric objects with oblique crossing angle is derived. The simulations' parameters are presented in Sec. III. Section IV provides the results of the simulations and discussions of the results and the limiting conditions. Finally, in Sec. V, conclusions of this paper are presented.

\section{THEORETICAL ANALYSIS}

\section{A. Forward transform}

It is well known that if a photon propagates through a medium with varying refractive index in space, the wavelength of the photon changes while its frequency remains constant. This does not happen if the medium is moving. In this case, the refractive index of the medium varies in both space and time. Thus, it changes the frequency and wavelength of photons that propagate in it.

When a plasma wakefield is generated in a plasma, the electron density variation gives different refractive indices at every position and time. If a laser pulse propagates in the wakefield, the frequency is shifted by the amount $[29,32]$

$$
\frac{\Delta \omega}{\omega_{0}} \approx-\frac{\omega_{p}^{2}}{2 \omega_{0}^{2}} \frac{c}{n_{0}} \int_{-\infty}^{\infty} \frac{\partial n}{\partial \zeta} \mathrm{d} t
$$

where $\Delta \omega / \omega_{0}$ is the fractional change of the frequency relative to its initial central frequency and $\omega_{p}$ represents the plasma frequency. The variable $\zeta=z-c t$ denotes the moving longitudinal position where $z$ and $t$ the position and time in the lab frame. The variables $n$ and $n_{0}$ are the electron density profile and its initial density, respectively, and $c$ is the speed of light in vacuum. The expression is integrated for the whole interaction time in the lab frame.

The configuration of a probe pulse at oblique angles to the plasma is shown in Fig. 1. The laser pulse propagates with angle $\theta$ and speed $v_{g}$, and crosses the wakefield which propagates in the $z$-direction with speed $u_{p}$. The laser pulse acts as a probe and is assumed to have very low power so that it leaves the wakefield relatively undisturbed. The goal in this section is to derive the relation between the frequency shift of a photon in the laser pulse and the electron density modulation profile of the wakefield. 


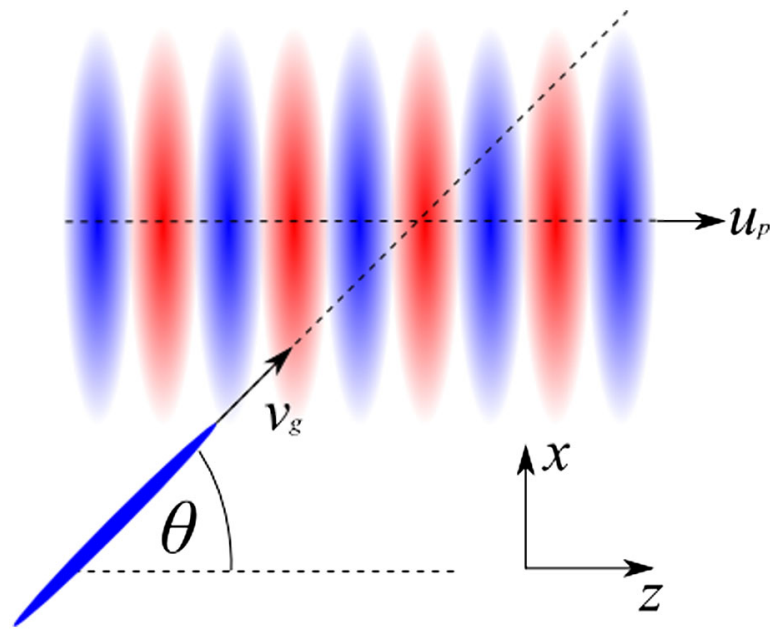

FIG. 1. The configuration considered in this paper: the laser probe pulse crosses the wakefield at an arbitrary oblique angle. The $y$-axis is pointed out of the page. By obtaining the phase or frequency modulation of the pulse, one can obtain the density modulation profile of the wakefield at the crossing point.

By taking $t=0$ when the photon is closest to the wakefield's axis, the position of the photon in the wakefield in cylindrical coordinates is

$$
\begin{aligned}
& r(t)=\left[\left(v_{g} t \sin \theta\right)^{2}+y^{2}\right]^{1 / 2}, \\
& x(t)=v_{g} t \sin \theta, \\
& \zeta(t)=\zeta_{0}+\left(v_{g} \cos \theta-u_{p}\right) t,
\end{aligned}
$$

where $r(t)$ denotes the distance from the wakefield's axis, $y$ is the shortest distance from the axis, and $\zeta_{0}$ represents the probe's longitudinal position when it is at the shortest distance from the axis.

In this case, the wakefield density profile is assumed to have a cylindrical symmetry. By defining $f(r, \zeta) \equiv$ $\left(-\omega_{p}^{2} c / 2 \omega_{0}^{2} n_{0}\right)(\partial n / \partial \zeta)$ and $F\left(y, \zeta_{0}\right) \equiv\left(\Delta \omega / \omega_{0}\right)\left(v_{g} \sin \theta\right)$, Eq. (1) can be written as

$$
F\left(y, \zeta_{0}\right)=v_{g} \sin \theta \int_{-\infty}^{\infty} f[r(t), \zeta(t)] \mathrm{d} t
$$

Using Eq. (2) and expanding $f[r(t), \zeta(t)]$ around $\zeta=\zeta_{0}$ using Taylor's series, the function can be written as

$$
f[r(t), \zeta(t)]=\sum_{j=0}^{\infty} \frac{\partial^{j} f}{\partial \zeta^{j}}\left[r(t), \zeta_{0}\right] \frac{\left[\left(v_{g} \cos \theta-u_{p}\right) t\right]^{j}}{j !} .
$$

Substituting $f(r, \zeta)$ from Eq. (4) to Eq. (3) and $t=$ $x / v_{g} \sin \theta$ from Eq. (2) yields

$$
F\left(y, \zeta_{0}\right)=\sum_{j=0}^{\infty} \frac{a^{j}}{j !} \int_{-\infty}^{\infty} \frac{\partial^{j} f}{\partial \zeta^{j}}\left(r, \zeta_{0}\right) x^{j} \mathrm{~d} x
$$

where $a \equiv\left[\left(\cos \theta-u_{p} / v_{g}\right) / \sin \theta\right]$ indicates how much the photon shifts horizontally relative to the wakefield.

The integration in Eq. (5) is an integration for $x$ while the function $f$ is expressed in $r$. From Eq. (2), variable $x$ can be substituted as $x= \pm \sqrt{r^{2}-y^{2}}$. The \pm sign on $x$ allows the integral to be split into two parts. The first integrates from $r=\infty$ to $r=y$ for negative $x$ and the second from $r=y$ to $r=\infty$ for positive $x$. Odd values of $j$ make the integrand signs for negative $x$ and positive $x$ the same. It therefore follows that the integrations for negative $x$ cancel the integrations for positive $x$ for odd values of $j$. It is important to note that this is not the case for even values of $j$, because the integrations for negative and positive $x$ have the same values. Rewriting Eq. (5) in terms of $r$ gives

$$
F\left(y, \zeta_{0}\right)=2 \sum_{j=0}^{\infty} \frac{a^{2 j}}{(2 j) !} \int_{y}^{\infty} \frac{\partial^{2 j} f}{\partial \zeta^{2 j}}\left(r, \zeta_{0}\right) \frac{\left(r^{2}-y^{2}\right)^{j}}{\sqrt{r^{2}-y^{2}}} r \mathrm{~d} r .
$$

This allows Eq. (6) to be calculated for known distributions of $f(r, \zeta)$. Further simplification can be made using a pseudodifferential operator with a Fourier transform [35]. One property of a Fourier transform is that one can transform $\partial^{j} f / \partial \zeta^{j}$ into $(i k)^{j} \tilde{f}$ where the tilde represents the Fourier transform of $f$, i.e., $\tilde{f}(r, k)=\int_{-\infty}^{\infty} f(r, \zeta) e^{-i k \zeta} \mathrm{d} \zeta$. By applying a Fourier transform on $f(r, \zeta)$ and $F\left(y, \zeta_{0}\right)$ in both the $\zeta$ and $\zeta_{0}$ directions, Eq. (6) is rewritten as

$$
\tilde{F}(y, k)=2 \int_{y}^{\infty} \sum_{j=0}^{\infty} \frac{(-1)^{j}}{(2 j) !}\left(k a \sqrt{r^{2}-y^{2}}\right)^{2 j} \frac{\tilde{f}(r, k) r \mathrm{~d} r}{\sqrt{r^{2}-y^{2}}} .
$$

The variable $\tilde{F}(y, k)$ represents the Fourier transformation of $F\left(y, \zeta_{0}\right)$, or $\tilde{F}(y, k)=\int_{-\infty}^{\infty} F\left(y, \zeta_{0}\right) e^{-i k \zeta_{0}} \mathrm{~d} \zeta_{0}$.

The Taylor's series of a cosine function is $\cos x=\sum_{j=0}^{\infty}(-1)^{j} x^{2 j} /(2 j)$ !, so the series terms in Eq. (7) can be substituted by a cosine function. Thus, the equation can be simplified as

$$
\tilde{F}(y, k)=2 \int_{y}^{\infty} \cos \left(k a \sqrt{r^{2}-y^{2}}\right) \frac{r \tilde{f}(r, k)}{\sqrt{r^{2}-y^{2}}} \mathrm{~d} r .
$$

This expression is similar to an Abel transformation [36-38] except that it contains a cosine factor in the integral. The zero value of the variable $a$ turns Eq. (8) into an Abel transform. It represents the special case where the probe pulse propagates perpendicularly relative to the wakefield.

To obtain $F\left(y, \zeta_{0}\right)$, Eq. (8) is simply transformed using the inverse Fourier transform. Most importantly, the equation applies for any case where measurements are made in 
cylindrically symmetric objects using nonperpendicular probes.

\section{B. Inverse transform}

When performing the measurement, one is mostly interested in obtaining the profile of $f(r, \zeta)$. To do this, one first measures $F\left(y, \zeta_{0}\right)$ and then one inverts it using the inverse transform of Eq. (8). This is

$$
\tilde{f}(r, k)=-\frac{1}{\pi} \int_{r}^{\infty} \frac{\partial \tilde{F}}{\partial y}(y, k) \frac{\cosh \left(k a \sqrt{y^{2}-r^{2}}\right)}{\sqrt{y^{2}-r^{2}}} \mathrm{~d} y .
$$

To verify Eq. (9), it is easier to start from Eq. (8) and rewrite it in the form of

$$
\tilde{F}(y, k)=-2 \int_{y}^{\infty} \frac{\sin \left(k a \sqrt{r^{2}-y^{2}}\right)}{k a} \frac{\partial \tilde{f}(r, k)}{\partial r} \mathrm{~d} r
$$

by using partial integration and assuming that $\lim _{r \rightarrow \infty} r \tilde{f}(r, k)=0$. Then taking the $y$-derivative of Eq. (10) yields

$$
\frac{\partial \tilde{F}(y, k)}{\partial y}=2 y \int_{y}^{\infty} \frac{\partial \tilde{f}(s, k)}{\partial s} \frac{\cos \left(k a \sqrt{s^{2}-y^{2}}\right)}{\sqrt{s^{2}-y^{2}}} \mathrm{~d} s .
$$

Substituting Eq. (11) into Eq. (9) gives

$$
\begin{aligned}
\tilde{f}(r, k)= & -\frac{2}{\pi} \int_{y=r}^{\infty} y \int_{s=y}^{\infty} \frac{\partial \tilde{f}(s, k)}{\partial s} \frac{\cos \left(k a \sqrt{s^{2}-y^{2}}\right)}{\sqrt{s^{2}-y^{2}}} \\
& \times \frac{\cosh \left(k a \sqrt{y^{2}-r^{2}}\right)}{\sqrt{y^{2}-r^{2}}} \mathrm{~d} s \mathrm{~d} y .
\end{aligned}
$$

Then by changing the order of integration, one obtains

$$
\begin{aligned}
\tilde{f}(r, k)= & -\frac{2}{\pi} \int_{s=r}^{\infty} \frac{\partial \tilde{f}(s, k)}{\partial s} \\
& \times \int_{y=r}^{s} y \frac{\cos \left(k a \sqrt{s^{2}-y^{2}}\right) \cosh \left(k a \sqrt{y^{2}-r^{2}}\right)}{\sqrt{s^{2}-y^{2}} \sqrt{y^{2}-r^{2}}} \\
& \times \mathrm{d} y \mathrm{~d} s .
\end{aligned}
$$

It can be shown that the $y$-integration on Eq. (12) is $\pi / 2$ and does not depend on $s, r, k$, and $a$ (see Appendix A). Thus, from Eq. (12), one can write

$$
\tilde{f}(r, k)=-\frac{2}{\pi} \int_{s=r}^{\infty} \frac{\partial \tilde{f}(s, k)}{\partial s} \frac{\pi}{2} \mathrm{~d} s=\tilde{f}(r, k),
$$

which confirms that Eq. (9) is the inverse of Eq. (8).

The inverse transformation in Eq. (9) can be implemented using 3-points Abel transformation with some modifications [39]. Details of the implementation technique are given in Appendix B.

\section{SIMULATION PARAMETERS}

Simulations using the OSIRIS 3D code [40-42] were performed. OSIRIS is a fully relativistic particle-in-cell code that can simulate plasma and electromagnetic waves and has been extensively benchmarked against experiments in laser plasma accelerators over the past two decades.

These simulations were performed to model realistic conditions expected in experiments and to check the accuracy of the measurements. In the baseline parameters of the three dimensional simulations, a cold plasma was used with a density of $n_{0}=2 \times 10^{19} \mathrm{~cm}^{-3}$. The simulation window contains $9750 \times 400 \times 300$ cells each with size of $(12 \times 120 \times 120) \mathrm{nm}^{3}$. One particle per cell was used and this was found to be sufficient for these purposes (the simulations were repeated using a larger number of particles per cell and gave similar results). A moving window simulation was deployed. Periodic boundary conditions were used in the transverse direction.

The driver of the wakefield was an electron beam which had a spherical Gaussian shape with $\sigma_{r}=4.4 \mu \mathrm{m}$ and peak density of $n_{e}=0.33 n_{0}$ to drive the wakefield amplitude around $\sim 0.53 n_{0}$. Each of the electrons in the driver beam propagated with momentum of $p / m_{e} c=45 \times 10^{3}$, where $m_{e}$ was the mass of an electron. The probe pulse was a plane wave with wavelength of $400 \mathrm{~nm}$, duration of $53 \mathrm{fs}$ and normalized intensity of $a_{0}=0.01$. The probe crossed the wakefield with an angle of $\theta=20^{\circ}$. In the simulation, 5 slices of the probe pulse were taken. The slices crossed the center of the wakefield at the propagation distance of $s=28,39,49,60$, and $67 \mu \mathrm{m}$ after the driver entered the plasma. These parameters were chosen to minimize the computational cost of the simulations. Each simulation took around $8000 \mathrm{CPU}$ hours to finish in average. Figure 2 illustrates the simulation and slicing of the electric field, taken from the actual results of the simulation.

To determine the accuracy of the measurement over some parameters, the density of the driver beam was varied from $0.1 n_{0}$ to $0.35 n_{0}$, the crossing angle from $25^{\circ}$ down to $5^{\circ}$, and the probe's wavelength from 260 to $800 \mathrm{~nm}$.

From the simulation results, the electron density profile was obtained, $n(r, \zeta)$, as a function of $r$, distance from its axis, and $\zeta$, longitudinal position in the moving frame. It is emphasized here that these profiles are labeled the "actual" density profiles. Besides obtaining the actual density profile, the frequency profiles of the sliced laser probe pulse, $\Delta \omega\left(y, \zeta_{0}\right)$, were also obtained as a function of the transverse position, $y$, and longitudinal position, $\zeta_{0}$. From this frequency profile and the known simulation parameters, the measured electron density modulation profiles were calculated from Eq. (9) and implemented using 3-points Abel transformation method as described in Appendix B. These density profiles are labeled the 

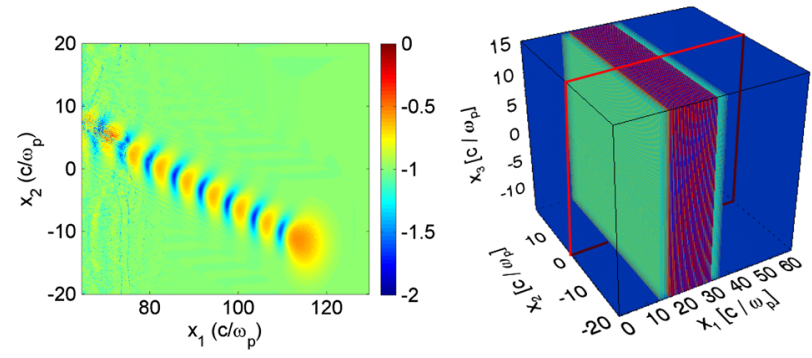

FIG. 2. Illustration of the simulation, taken from the actual results of the simulation. The left picture show the sliced wakefield in the electron density profile at $x_{3}=0$. The right picture illustrates the initial electric field profile of the probe pulse. The red line on the right picture shows one of five planes where the slice of the electric field crosses the wakefield at the propagation distance $s=28 \mu \mathrm{m}$ after the driver entered the plasma. The axes $x_{1}, x_{2}$, and $x_{3}$ in this illustration are different from the axes $x, y, z$ in the previous figure where in this illustration the laser probe pulse propagate parallel with $x_{1}$-axis. While in Fig. 1, the probe pulse propagates not parallel to any axis.

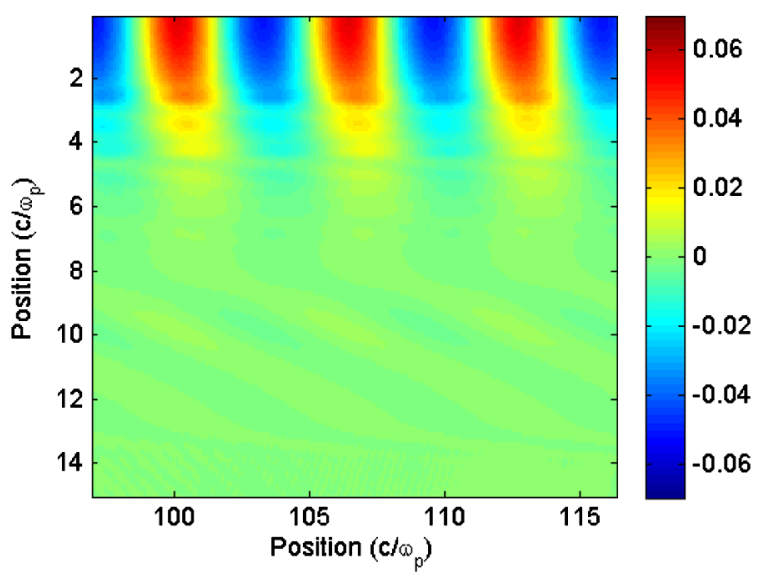

(a)

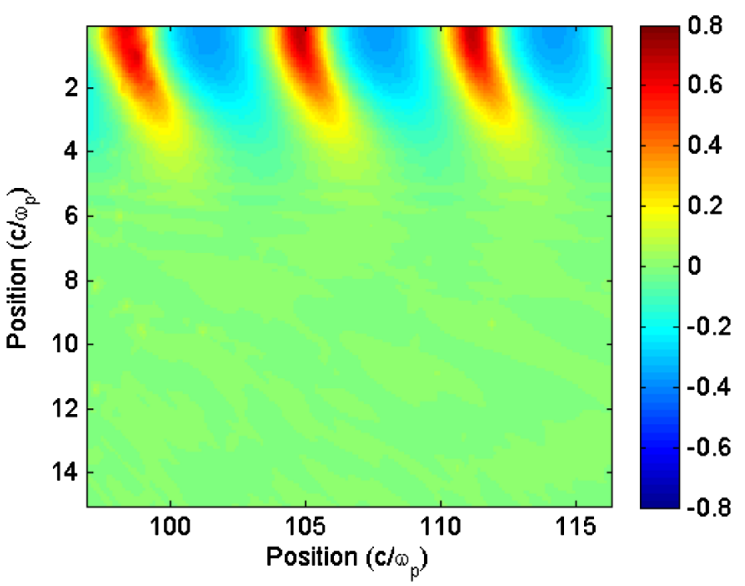

(c) "measured" profiles. The actual and the measured density modulation profiles are compared to see how well those values agree.

To extract the frequency profile of the laser probe pulse in the simulation, a Wigner transform was used, as described in Ref. [33]. This gives flexibility in the choice of $2 \mathrm{D}$ phase or frequency extraction methods in real experiments. These include single-shot supercontinuum spectral interferometry $[21,22,25]$, frequency domain interferometry [43,44], SPIDER [45], etc. Therefore, analysis of the accuracy and precision in real experiments need to be combined with error analysis from the chosen method.

\section{RESULTS AND DISCUSSION}

\section{A. Results of measurement simulations}

In the simulations, the wakefield amplitude did not significantly change during the interaction with the probe pulse. Also, the amplitude of frequency modulation of the laser pulse is not significantly different between the slices

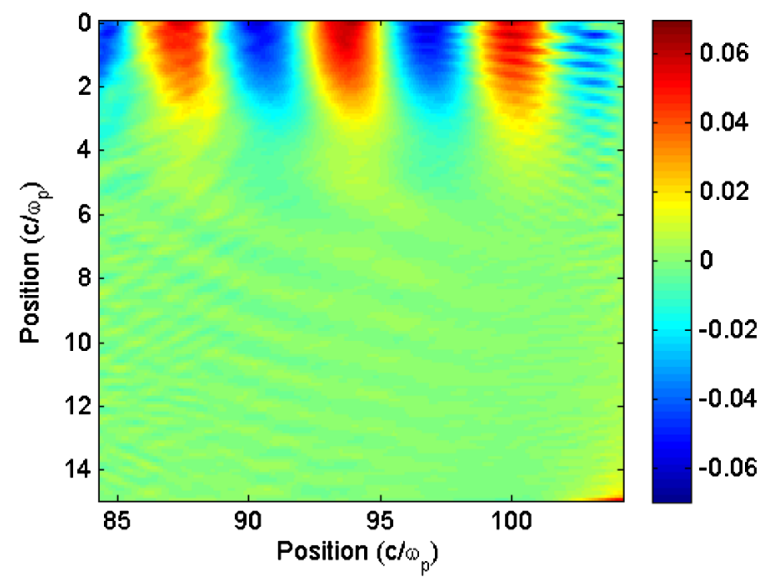

(b)

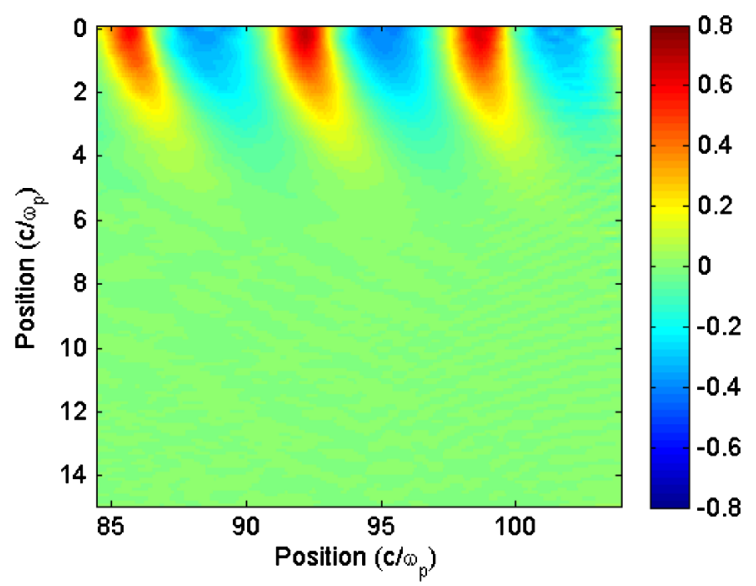

(d)

FIG. 3. Shape of the electron density modulation profile as a function of position (a) obtained directly from the simulation results and (b) calculated from the probe's frequency profile for a linear wakefield and (c)-(d) for a nonlinear wakefield. The distortion at the front and at the back on (d) is because the intensity of the pulse at that point is low, hence reduces the accuracy of the retrieved frequency. 


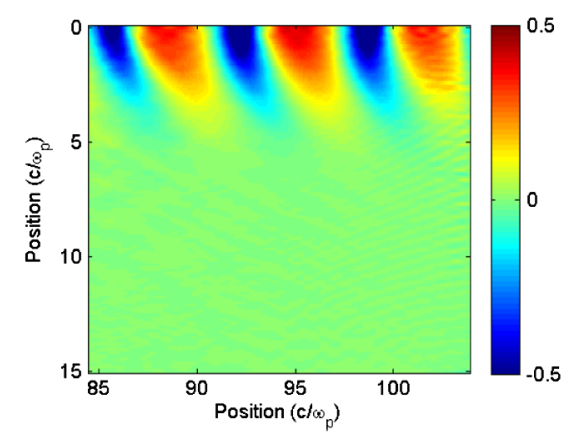

(a)

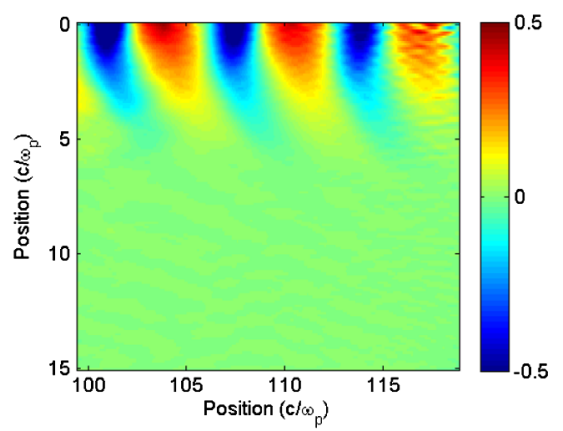

(b)

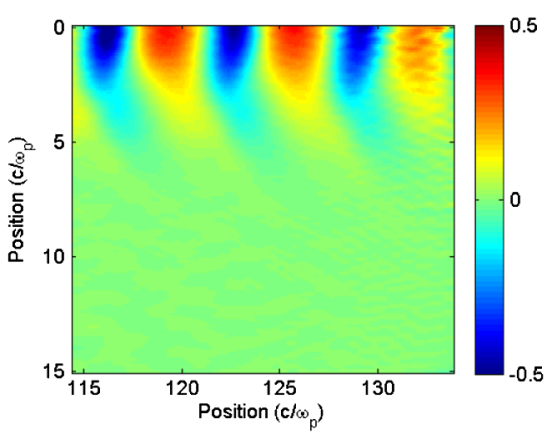

(c)

FIG. 4. Evolution of the measured density modulation profile resulting from diffraction after the interaction with the wakefield. (a) The profile when it just finishes interacting with the wakefield, (b) when it propagates $18 \mu \mathrm{m}$ from the previous picture and (c) after it propagates $36 \mu \mathrm{m}$ from the first picture. The shape of the modulated profile is changing and decreases in amplitude as it propagates further from the interaction point.

taken along the plasma column. The 2D frequency profile of the slice of the laser pulse that crosses the center of the wakefield at $s=28 \mu \mathrm{m}$ was obtained shortly after it had interacted with the wakefield for the cases of small and large wakefield amplitudes. Figure 3 shows the comparison between the measured electron density modulation profiles from the laser pulse and the actual density modulation profiles for both cases.

As seen in Fig. 3, the measured electron density profiles share similar shapes with the actual density profiles shortly after the probe pulse crosses the wakefield. However, as the pulse propagates, the measured density profile from the probe pulse changes its shape. The amplitude of the modulation also decreases, as seen in Fig. 4. Therefore, measuring the density profile after the laser pulse propagates some distance could lead to inaccurate measurements. This is an unwanted diffraction effect and can be mitigated in real experiments using $4 f$ image relaying setups [46]. Another unwanted effect is dispersion which can be eluded by avoiding transmissive optics in the experiment.

Before the diffraction effect on the measured profile becomes significant, a comparison was made between the peaks and troughs values of the measured and actual density modulation profiles. Figure 5 shows the peaks and troughs values of the actual and measured density modulation profiles from 5 slices along the plasma column. These actual and measured values show excellent agreement.

In order to do a quantitative comparison for various driver beam densities, crossing angles, and frequencies of the probe pulse, the average values of the amplitudes of the measured and actual density modulation profiles were computed for each case. The amplitude here was defined as half of the difference between the peak and the trough values. The relative error between the actual and the measured values were also computed for each set of parameters.

For various driver beam densities, the comparison between the actual and the measured values is shown in
Fig. 6(a) and their relative errors are in Fig. 6(b). The figures show that the relative errors of the measurement do not have any particular trend for the driver beam density less than or equal to $0.35 n_{0}$. This is where the wakefield
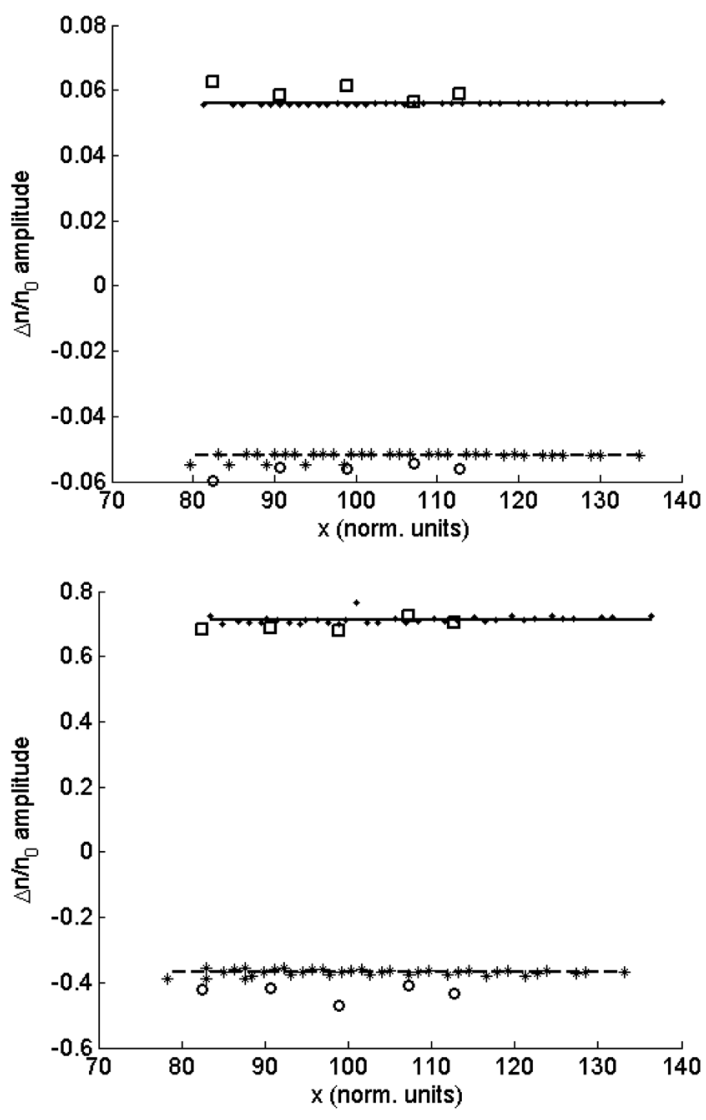

FIG. 5. Comparison between actual density modulation profile peaks (dots) and troughs (stars) with the measured density modulation profile (squares and circles). Solid and dash lines show the average values of the peaks and troughs of the actual density modulation profile, respectively. The top picture shows the comparison for small wakefield amplitude and the bottom picture for large wakefield amplitude. 


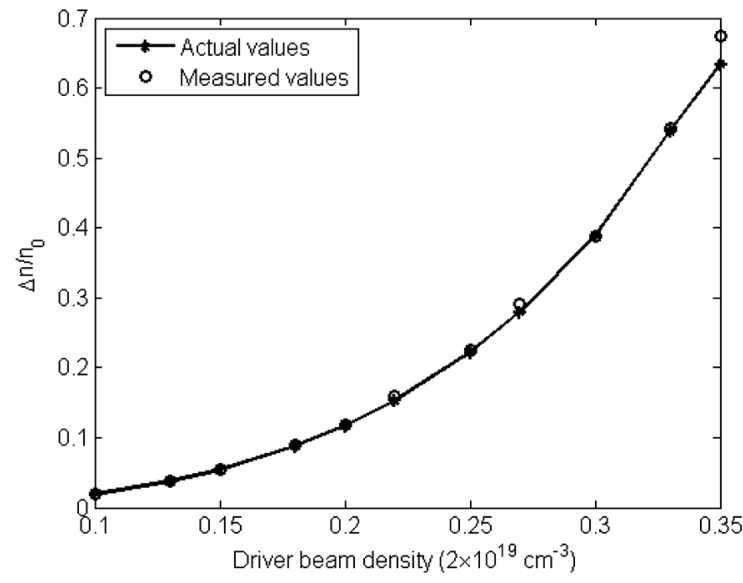

(a)

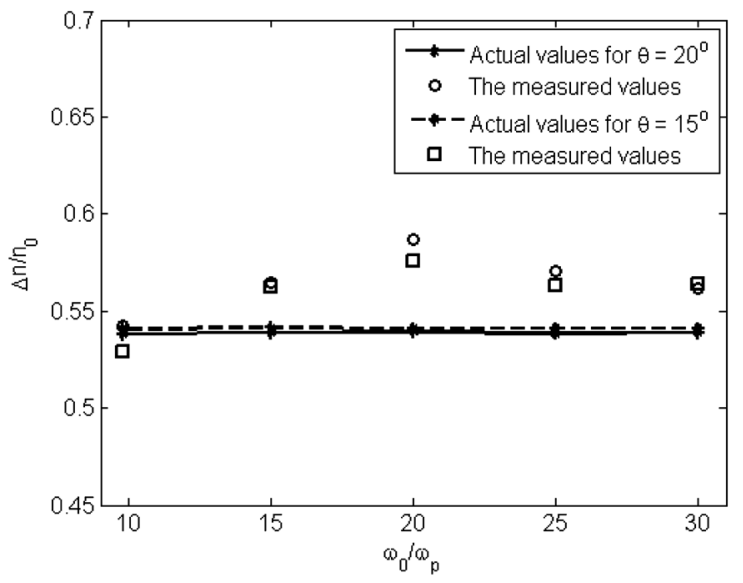

(c)

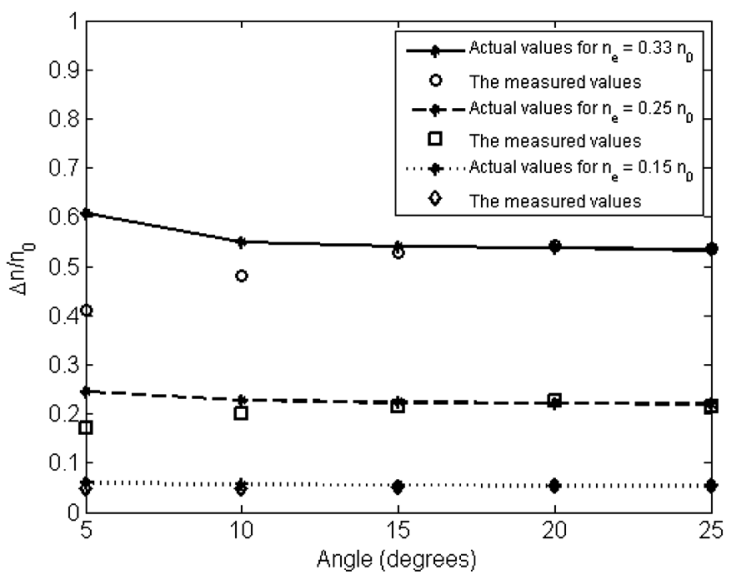

(e)

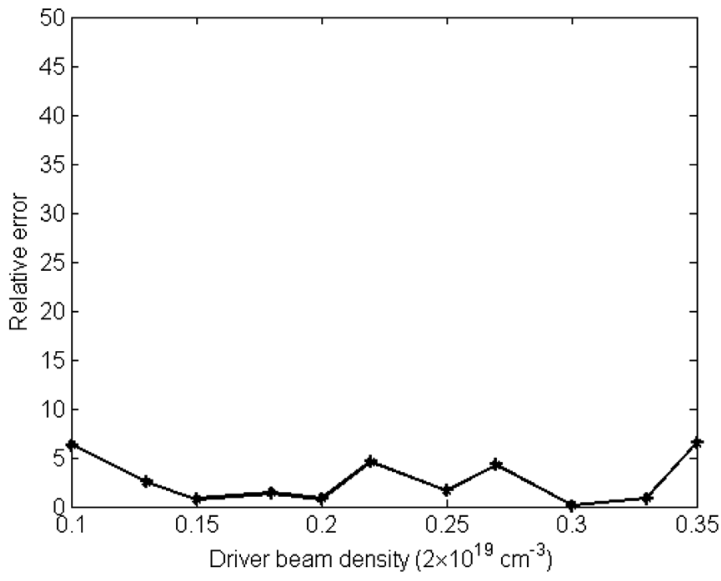

(b)

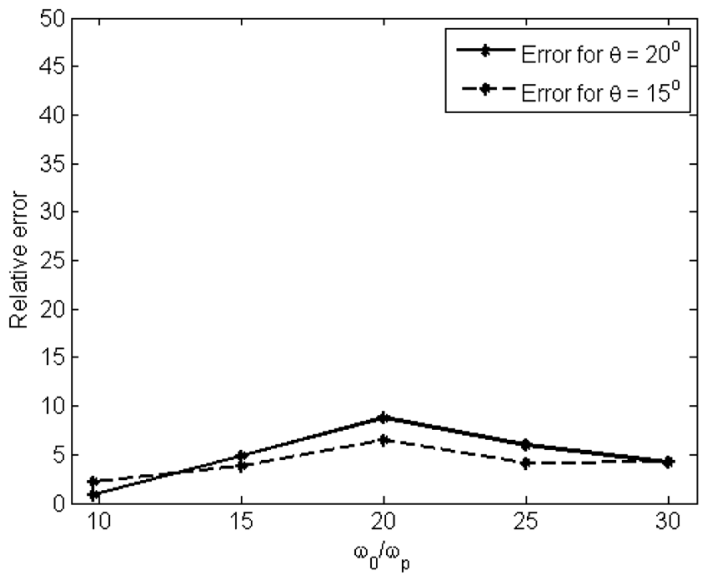

(d)

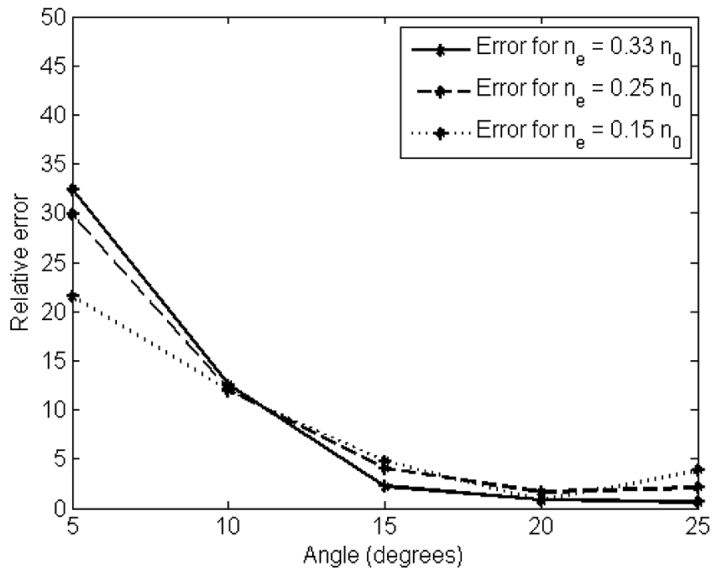

(f)

FIG. 6. Comparison of the measured and actual amplitude of the density modulation profiles for (a) various driver beam's densities and (b) the relative errors; (c) various probe's frequencies and (d) the errors; (e) various angles and (f) the errors. The measured values are calculated from the frequency modulation profiles of the laser probe pulse using Eq. (9). The actual values are obtained directly from the electron density modulation profiles in the simulations. 
amplitude reaches $\sim 0.64 n_{0}$. It should be noted that none of these errors exceed $10 \%$.

The next comparison is varying the probe's wavelength from 260 to $800 \mathrm{~nm}$, while the other parameters remain the same as the baseline parameters. The comparison values and the relative errors are shown in Figs. 6(c) and (d). These indicate that the error values do not change significantly as the probe's frequency increases. Therefore the error is independent of the probe's wavelength.

Another comparison was also done to check the dependency of the crossing angle with the measured values using $800 \mathrm{~nm}$ probe. This comparison was made with three different values of driver beam density and was designed to cover the linear and nonlinear regimes of the wakefield. The results of this comparison and the errors are shown in Figs. 6(e) and (f). For $n_{e}=0.15 n_{0}$ and $n_{e}=0.25 n_{0}$, decreasing the crossing angle increases the relative error. This is because when the angle decreases, the interaction length between the wakefield and the probe pulse becomes longer. If the interaction length is comparable to the diffraction length of the modulated part of the pulse, the frequency modulation's amplitude of the laser pulse decreases because of diffraction, hence increases the relative error. For all cases in Figs. 6(e) and (f), most of the relative errors are less than $10 \%$, except when the crossing angle $\theta \leq 10^{\circ}$ because of diffraction. This diffraction effect will be discussed in the next subsection.

\section{B. Measurement constraints}

A photon acceleration diagnostic with oblique angle has several constraints and limitations that can make the measurement results inaccurate. In the previous paper [33], several limitations in doing the measurement using photon acceleration with a copropagating probe pulse have been discussed. These include maximum propagation distance to avoid photon trapping and maximum intensity of the probe pulse to avoid stimulated Raman scattering. More details can be found in Ref. [33].

In this oblique crossing angle setting, there are more additional constraints. Some of those are apparent in the results provided in the previous subsection. In this subsection, these new constraints are discussed in greater detail to provide a realistic guide to realize this measurement technique in the laboratory.

\section{Diffraction}

Consider the electric field of the probe as a function of position, $U_{0}(\mathbf{r}, t)=\left|U_{0}(\mathbf{r}, t)\right| e^{i \mathbf{k} \cdot \mathbf{r}-i \omega_{0} t}$. As it crosses the wakefield, the probe will experience a phase and frequency modulation. Thus, the modulated electric field profile can now be written as $U(\mathbf{r}, t)=U_{0}(\mathbf{r}, t) e^{i \Delta \phi(\mathbf{r}, t)}$, where $\Delta \phi(\mathbf{r}, t)$ is the phase modulation resulting from laserwakefield interaction. If the modulation is small enough, it can be approximated as

$$
U(\mathbf{r}, t) \approx U_{0}(\mathbf{r}, t)+i U_{0}(\mathbf{r}, t) \Delta \phi(\mathbf{r}, t) .
$$

From the equation above, the modulated part of the probe can be regarded as a new wave propagating in the same direction with approximately same frequency, but with amplitude profile of $\left|U_{0}(\mathbf{r}, t)\right||\Delta \phi(\mathbf{r}, t)|$.

In the photon acceleration case, when a very wide probe crosses a small wakefield, the probe pulse will have a modulated part with a size approximately the same as that of the wakefield. Because the modulated part has the smaller size, it will diffract faster than the unperturbed part of the probe. This diffraction effect reduces the phase and frequency modulation of the pulse if the interaction length is larger than its diffraction length. In order to minimize the diffraction effect, the crossing angle, $\theta$, should be large enough to keep the interaction length short, or

$$
\sin \theta \gtrsim \frac{\lambda_{0}}{\sqrt{\pi} r_{p}}
$$

where $\lambda_{0}$ is the probe's wavelength and $r_{p}$ is the wakefield's radius.

In the simulated cases, the crossing angle should be $\theta \gtrsim 8^{\circ}$. This explains why the relative errors start increasing when $\theta=10^{\circ}$ and increase significantly when the crossing angle is set to be $5^{\circ}$ for any simulated driver densities as shown in Fig. 6(f).

\section{Error dependence on angle}

It is interesting to see how the measurement error is related to the angle of incidence. As seen later in this subsection, the error analysis on the angle can set the upper limit of the crossing angle.

In order to simplify the analysis, it is assumed that the wakefield has a perfect sinusoidal in the longitudinal position and a Gaussian profile in the transverse position. Although this is not the case for nonlinear wakefield and some other cases (e.g., non-Gaussian transverse profile of the driver), it is still useful to quantify the error. For this simplified case, the frequency shift of the laser pulse has a similar profile and can be written as

$$
\frac{\Delta \omega}{\omega_{0}}=\left(\frac{\Delta \omega}{\omega_{0}}\right)_{\max } \sin \left(\omega_{p} \zeta_{0} / c\right) e^{-y^{2} / r_{p}^{2}}
$$

where $r_{p}$ represents the radius of the wakefield at which the value drops to $1 / e$ of the axial value. Other variables are as introduced before. Applying the inverse transformation in Eq. (9), the wakefield density is obtained as

$$
\frac{\Delta n}{n_{0}}=\left(\frac{\Delta n}{n_{0}}\right)_{\max } \sin \left(\omega_{p} \zeta / c\right) e^{-r^{2} / r_{p}^{2}}
$$

where 


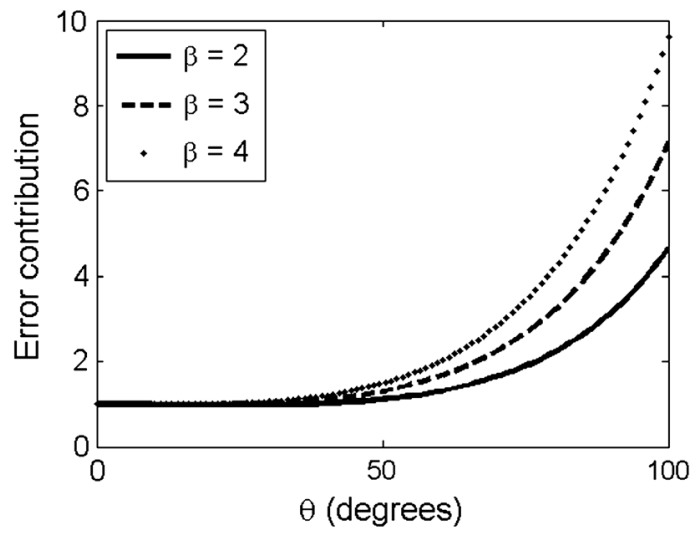

FIG. 7. Relative error contribution from the angle to the wakefield density measurement for various values of $\beta \equiv$ $\omega_{p}^{2} r_{p}^{2} / 2 c^{2}$ in Eq. (20).

$$
\left(\frac{\Delta n}{n_{0}}\right)_{\max }=-\frac{2}{\sqrt{\pi}} \frac{\omega_{0}^{2} c v_{g} \sin \theta}{\omega_{p}^{3} u_{p} r_{p}} e^{\frac{1}{4} \omega_{p}^{2} a^{2} r_{p}^{2} / c^{2}}\left(\frac{\Delta \omega}{\omega_{0}}\right)_{\max } .
$$

Notice that the oblique crossing angle raises an exponential effect in $\left(\Delta n / n_{0}\right)_{\max }$ for large absolute value of $a$. Defining $f_{m} \equiv\left(\Delta n / n_{0}\right)_{\max }$ makes the equation simpler.

If there is an error in quantifying the angle, the contribution of this error from Eq. (18) is

$$
\frac{\delta f_{m}}{f_{m}}=\left(\frac{\omega_{p}^{2} r_{p}^{2}}{2 c^{2}} \frac{\cos \theta-\frac{u_{p}}{v_{g}} \frac{u_{p}}{v_{g}} \cos \theta-1}{\sin \theta} \theta+\frac{\theta}{\sin \theta}\right) \frac{\delta \theta}{\theta} .
$$

In most cases of plasma wakefield accelerators, both the probe and the driver travel with speed near the speed of light, so it can be assumed that $u_{p} / v_{g} \approx 1$. Thus, Eq. (19) can be written in a simpler form,

$$
\frac{\delta f_{m}}{f_{m}} \approx\left[\frac{\theta}{\tan \theta}+\frac{\omega_{p}^{2} r_{p}^{2}}{2 c^{2}}\left(\frac{1-\cos \theta}{\sin \theta}\right)^{2} \theta\right] \frac{\delta \theta}{\theta} .
$$

Figure 7 shows how the error contribution from the angle [the term inside the right angle brackets in Eq. (20)] grows as the crossing angle grows for various values of $\beta \equiv \omega_{p}^{2} r_{p}^{2} / 2 c^{2}$. The graph shows that the error contribution grows faster as the crossing angle increases.

From the graph, one can choose the maximum angle that could give reasonable error contribution from the angle. One possible choice of maximum angle is when the error contribution from the angle reaches 2 . Other values can be chosen with trade-off between error and the maximum angle. For the simulated cases in this paper, the error from quantifying the angle is doubled when $\theta \approx 60^{\circ}$.

\section{CONCLUSIONS}

The first quantitative theoretical and simulation studies of photon acceleration diagnostics with oblique crossing angle on plasma wakefield have been presented. A small intensity probe pulse crosses the wakefield at certain positions with a defined angle of incidence. By choosing the interaction point between the probe and the wakefield, it has been shown here that one can obtain an image of the plasma wakefield density modulation profile at that point. Therefore, it is possible to detect the evolution of plasma wakefield along the propagation distance.

Results from our simulated measurements show that the density modulation measured from the frequency modulation profiles of the probe pulse agrees with the actual electron density modulation profiles to within $10 \%$ relative error. The constraints that set the lower and upper limit of the crossing angle to minimize errors to obtain electron density modulation profiles are also discussed in this paper. These are the diffraction effect and error dependency analysis on the crossing angle. By considering the constraints, one can determine the optimal parameters in both laser and beam-driven wakefield accelerators to extract the density modulation profile as a function of position along the plasma column.

\section{ACKNOWLEDGMENTS}

The authors would like to acknowledge the support from the plasma physics HEC Consortium EPSRC Grant No. EP/L000237/1, as well as the Central Laser Facility and the Scientific Computing Department at the Rutherford Appleton Laboratory for the use of SCARF-LEXICON computer cluster. We would also like to thank ARCHER UK National Supercomputing Service for the use of the computing service. We also wish to thank the UCLA/IST OSIRIS consortium for the use of OSIRIS and also to the Science and Technology Facilities Council for its support to AWAKE-UK (Grants No. ST/K002244/1 and No. ST/ M007375/1). One of the authors (M. F. Kasim) would like to thank Indonesian Endowment Fund for Education for its support. M. C. L. thanks the Royal Society Newton International Fellowship for support. J. S. and N. R. acknowledge the support from Engineering and Physical Sciences Research Council. M. W. acknowledges the support of DESY, Hamburg, and the Alexander von Humboldt Foundation. The work is part of EuCARD-2, partly funded by the European Commission, GA 312453. The authors gratefully acknowledge the support of all of the staff of STFC's Central Laser Facility in the execution of this work.

\section{APPENDIX A:}

In this part, it will be proven that the $y$-integral on Eq. (12) is constant, i.e.,

$$
I=\int_{y=r}^{s} y \frac{\cos \left(k a \sqrt{s^{2}-y^{2}}\right) \cosh \left(k a \sqrt{y^{2}-r^{2}}\right)}{\sqrt{s^{2}-y^{2}} \sqrt{y^{2}-r^{2}}} \mathrm{~d} y=\frac{\pi}{2} .
$$


Substituting $x^{2} \equiv y^{2}-r^{2}$ and thus $x \mathrm{~d} x=y \mathrm{~d} y$ into the equation above gives

$$
I=\int_{x=0}^{\sqrt{s^{2}-r^{2}}} \frac{\cos \left[k a \sqrt{\left(s^{2}-r^{2}\right)-x^{2}}\right] \cosh (k a x)}{\sqrt{\left(s^{2}-r^{2}\right)-x^{2}}} \mathrm{~d} x .
$$

Now substitute $x=\sqrt{s^{2}-r^{2}} \sin \theta$ to the equation above so it can be simplified into

$$
I=\int_{0}^{\pi / 2} \cos (b \cos \theta) \cosh (b \sin \theta) \mathrm{d} \theta,
$$

where $b \equiv k a \sqrt{s^{2}-r^{2}}$. Using the representation of cosine and hyperbolic cosine in exponential form, the integral can be further simplified into

$$
I=\frac{1}{2} \int_{-\pi / 2}^{\pi / 2} \cos \left(b e^{i \theta}\right) \mathrm{d} \theta .
$$

Taking the derivative of $I$ with respect to $b$ yields

$$
\begin{aligned}
\frac{\partial I}{\partial b} & =-\frac{1}{2} \int_{-\pi / 2}^{\pi / 2} e^{i \theta} \sin \left(b e^{i \theta}\right) \mathrm{d} \theta \\
& =\left.\frac{1}{2 i b} \cos \left(b e^{i \theta}\right)\right|_{-\pi / 2} ^{\pi / 2}=0
\end{aligned}
$$

Zero derivative of $I$ means that value of $I$ does not depend on $b$. Thus, the integral can be evaluated at limit $b \rightarrow 0$ to get the value of the integral,

$$
I=\lim _{b \rightarrow 0} \frac{1}{2} \int_{-\pi / 2}^{\pi / 2} \cos \left(b e^{i \theta}\right) \mathrm{d} \theta=\frac{\pi}{2}
$$

as used in Eq. (13).

\section{APPENDIX B:}

The inverse of modified Abel transformation in Eq. (9) contains singularity when $y=r$. The modified 3-points Abel inversion can be employed to overcome this problem in the implementation.

First, the integral term in Eq. (9) is split into several integrals with spacing $\Delta r$,

$$
f\left(r_{j}\right)=-\frac{1}{\pi} \sum_{i \geq j} \int_{y_{i 0}}^{y_{i f}} F^{\prime}(y) \frac{\cosh \left(k a \sqrt{y^{2}-r_{j}^{2}}\right)}{\sqrt{y^{2}-r_{j}^{2}}} \mathrm{~d} y,
$$

where $r_{j}=j \Delta r, \quad y_{i f}=i \Delta r+\Delta r / 2, \quad y_{i 0}=i \Delta r+g_{i j} \Delta r$, and

$$
g_{i j}= \begin{cases}0 & \text { if } i=j \\ -1 / 2 & \text { otherwise }\end{cases}
$$

Note that the second argument, $k$, and the tilde hat from $\tilde{f}(r, k)$ and $\tilde{F}(y, k)$ in Eq. (9) are dropped for the sake of simplicity. The term $\partial \tilde{F} / \partial y$ is denoted by $F^{\prime}$.

Substituting $r_{j}=j \Delta r$ and $y=i \Delta r+\delta \Delta r$ to the integral gives

$f\left(r_{j}\right)$

$$
=-\frac{1}{\pi} \sum_{i \geq j} \int_{g_{i j}}^{1 / 2} \frac{F^{\prime}(i \Delta r+\delta \Delta r) \cosh \left(k a \Delta r \sqrt{(i+\delta)^{2}-j^{2}}\right)}{\sqrt{(i+\delta)^{2}-j^{2}}} \mathrm{~d} \delta .
$$

The term $F^{\prime}(i \Delta r+\delta \Delta r)$ and the hyperbolic cosine term can be expanded using Taylor series around $i \Delta r$ to the first order. The expansions are

$$
F^{\prime}(i \Delta r+\delta \Delta r) \approx F^{\prime}(i \Delta r)+F^{\prime \prime}(i \Delta r) \delta \Delta r
$$

and

$$
\cosh \left(k a \Delta r \sqrt{(i+\delta)^{2}-j^{2}}\right)=C_{i j}+S_{i j} \delta
$$

where $F^{\prime \prime}$ is the second derivative of the function $F$ relative to $y, C_{i j}=\cosh \left(k a \Delta r \sqrt{i^{2}-j^{2}}\right)$, and $S_{i j}=$ $\sinh \left(k a \Delta r \sqrt{i^{2}-j^{2}}\right) k a i \Delta r / \sqrt{i^{2}-j^{2}}$.

Substituting these two expanded terms in the integral and keeping only the first order terms of $\delta$ leaves us an analytically integrable expression. Evaluating the integral analytically leaves us the equation below,

$$
f\left(r_{j}\right)=-\frac{1}{\pi} \sum_{i \geq j}\left\{F_{i}^{\prime} C_{i j} B_{i j}^{(0)}+\left[F_{i}^{\prime \prime} \Delta r C_{i j}+F_{i}^{\prime} S_{i j}\right] B_{i j}^{(1)}\right\},
$$

where $F_{i}$ is a shorthand for $F(i \Delta r)$ and

$$
\begin{aligned}
& B_{i j}^{(0)}= \begin{cases}0 & ; i=j=0 \quad \text { or } \quad i<j \\
\ln \left[\frac{2 i+1+\sqrt{(2 i+1)^{2}-4 j^{2}}}{2 i}\right] & ; i=j \neq 0 \\
\ln \left[\frac{2 i+1+\sqrt{(2 i+1)^{2}-4 j^{2}}}{2 i-1+\sqrt{(2 i-1)^{2}-4 j^{2}}}\right] & ; i>j\end{cases} \\
& B_{i j}^{(1)}= \begin{cases}0 & ; i=j=0 \quad \text { or } \quad i<j \\
D_{i j}^{+}-i B_{i j}^{(0)} & ; i=j \neq 0 \\
D_{i j}^{+}-D_{i j}^{-}-i B_{i j}^{(0)} & ; i>j,\end{cases}
\end{aligned}
$$

with $D_{i j}^{ \pm} \equiv \sqrt{(i \pm 1 / 2)^{2}-j^{2}}$.

In quadratic interpolation, the first and second derivative of the function $F$ can be expressed as

$$
\begin{aligned}
F_{i}^{\prime} & =\left(F_{i+1}-F_{i-1}\right) / 2 \Delta r \\
F_{i}^{\prime \prime} & =\left(F_{i+1}+F_{i-1}-2 F_{i}\right) / \Delta r^{2} .
\end{aligned}
$$

Inserting this $F_{i}^{\prime}$ and $F_{i}^{\prime \prime}$ terms yields 


$$
\begin{aligned}
f\left(r_{j}\right)= & -\frac{1}{\pi \Delta r} \sum_{i \geq j}\left\{F_{i}\left[-2 C_{i j} B_{i j}^{(1)}\right]\right. \\
& +F_{i-1}\left[-\frac{1}{2} C_{i j} B_{i j}^{(0)}+C_{i j} B_{i j}^{(1)}-\frac{1}{2} S_{i j} B_{i j}^{(1)}\right] \\
& \left.+F_{i+1}\left[\frac{1}{2} C_{i j} B_{i j}^{(0)}+C_{i j} B_{i j}^{(1)}+\frac{1}{2} S_{i j} B_{i j}^{(1)}\right]\right\} .
\end{aligned}
$$

The 3-points Abel inversion method is similar with the technique described in Ref. [39], except that the hyperbolic cosine term is also expanded in this method.

Another problem that may arise in the implementation is that the hyperbolic cosine term can amplify the high frequency noise. This problem can be solved simply by choosing a cutoff value, $k_{c}$ and set $k=0$ when $k \geq k_{c}$.

[1] T. Tajima and J. M. Dawson, Phys. Rev. Lett. 43, 267 (1979).

[2] A. Modena, Z. Najmudin, A. E. Dangor, C. E. Clayton, K. A. Marsh, C. Joshi, V. Malka, C. B. Darrow, C. Danson, D. Neely et al., Nature (London) 377, 606 (1995).

[3] C. Joshi, W. B. Mori, T. Katsouleas, J. M. Dawson, J. M. Kindel, and D. W. Forslund, Nature (London) 311, 525 (1984).

[4] S. P. D. Mangles, C. D. Murphy, Z. Najmudin, A. G. R. Thomas, J. L. Collier, A. E. Dangor, E. J. Divall, P. S. Foster, J. G. Gallacher, C. J. Hooker et al., Nature (London) 431, 535 (2004).

[5] C. G. R. Geddes, Cs. Toth, J. van Tilborg, E. Esarey, C. B. Schroeder, D. Bruhwiler, C. Nieter, J. Cary, and W. P. Leemans, Nature (London) 431, 538 (2004).

[6] J. Faure, Y. Glinec, A. Pukhov, S. Kiselev, S. Gordienko, E. Lefebvre, J.-P. Rousseau, F. Burgy, and V. Malka, Nature (London) 431, 541 (2004).

[7] W. P. Leemans, B. Nagler, A. J. Gonsalves, Cs. Tóth, K. Nakamura, C. G. R. Geddes, E. Esarey, C. B. Schroeder, and S. M. Hooker, Nat. Phys. 2, 696 (2006).

[8] V. Malka, S. Fritzler, E. Lefebvre, M.-M. Aleonard, F. Burgy, J.-P. Chambaret, J.-F. Chemin, K. Krushelnick, G. Malka, S. P. D. Mangles et al., Science 298, 1596 (2002).

[9] F. Amiranoff, S. Baton, D. Bernard, B. Cros, D. Descamps, F. Dorchies, F. Jacquet, V. Malka, J. R. Marquès, G. Matthieussent et al., Phys. Rev. Lett. 81, 995 (1998).

[10] Y. Kitagawa, T. Matsumoto, T. Minamihata, K. Sawai, K. Matsuo, K. Mima, K. Nishihara, H. Azechi, K. A. Tanaka, H. Takabe et al., Phys. Rev. Lett. 68, 48 (1992).

[11] C. E. Clayton, K. A. Marsh, A. Dyson, M. Everett, A. Lal, W. P. Leemans, R. Williams, and C. Joshi, Phys. Rev. Lett. 70, 37 (1993).

[12] F. Amiranoff, D. Bernard, B. Cros, F. Jacquet, G. Matthieussent, P. Miné, P. Mora, J. Morillo, F. Moulin, A. E. Specka et al., Phys. Rev. Lett. 74, 5220 (1995).

[13] J. B. Rosenzweig, D. B. Cline, B. Cole, H. Figueroa, W. Gai, R. Konecny, J. Norem, P. Schoessow, and J. Simpson, Phys. Rev. Lett. 61, 98 (1988).
[14] I. Blumenfeld, C. E. Clayton, F.-J. Decker, M. J. Hogan, C. Huang, R. Ischebeck, R. Iverson, C. Joshi, T. Katsouleas, N. Kirby et al., Nature (London) 445, 741 (2007).

[15] P. Muggli, B. E. Blue, C. E. Clayton, S. Deng, F.-J. Decker, M. J. Hogan, C. Huang, R. Iverson, C. Joshi, T. C. Katsouleas et al., Phys. Rev. Lett. 93, 014802 (2004).

[16] A. Caldwell, K. Lotov, A. Pukhov, and F. Simon, Nat. Phys. 5, 363 (2009).

[17] N. Kumar, A. Pukhov, and K. Lotov, Phys. Rev. Lett. 104, 255003 (2010).

[18] M. Litos, E. Adli, W. An, C. I. Clarke, C. E. Clayton, S. Corde, J. P. Delahaye, R. J. England, A. S. Fisher, J. Frederico et al., Nature (London) 515, 92 (2014).

[19] E. Esarey, C. B. Schroeder, and W. P. Leemans, Rev. Mod. Phys. 81, 1229 (2009).

[20] D. Gordon, K. C. Tzeng, C. E. Clayton, A. E. Dangor, V. Malka, K. A. Marsh, A. Modena, W. B. Mori, P. Muggli, Z. Najmudin et al., Phys. Rev. Lett. 80, 2133 (1998).

[21] N. H. Matlis, S. Reed, S. S. Bulanov, V. Chvykov, G. Kalintchenko, T. Matsuoka, P. Rousseau, V. Yanovsky, A. Maximchuk, S. Kalmykov et al., Nat. Phys. 2, 749 (2006).

[22] K. Y. Kim, I. Alexeev, and H. M. Milchberg, Appl. Phys. Lett. 81, 4124 (2002).

[23] Z. Li, R. Zgadzaj, X. Wang, Y.-Y. Chang, and M. C. Downer, Nat. Commun. 5, 3085 (2014).

[24] A. Maksimchuk, S. Reed, S. S. Bulanov, V. Chvykov, G. Kalintchenko, T. Matsuoka, C. McGuffey, G. Mourou, N. Naumova, J. Nees et al., Phys. Plasmas 15, 056703 (2008).

[25] Z. Li, H.-E. Tsai, X. Zhang, C.-H. Pai, Y.-Y. Chang, R. Zgadzaj, X. Wang, V. Khudik, G. Shvets, and M. C. Downer, Phys. Rev. Lett. 113, 085001 (2014).

[26] A. Buck, M. Nicolai, K. Schmid, C. M. S. Sears, A. Sävert, J. M. Mikhailova, F. Krausz, M. C. Kaluza, and L. Veisz, Nat. Phys. 7, 543 (2011).

[27] A. Sävert, S. P. D. Mangles, M. Schnell, J. M. Cole, M. Nicolai, M. Reuter, M. B. Schwab, M. Möller, K. Poder, O. Jäckel et al., arXiv:1402.3052.

[28] S. C. Wilks, J. M. Dawson, W. B. Mori, T. Katsouleas, and M. E. Jones, Phys. Rev. Lett. 62, 2600 (1989).

[29] J. M. Dias, L. Oliveira e Silva, and J. T. Mendonça, Phys. Rev. ST Accel. Beams 1, 031301 (1998).

[30] C. D. Murphy, R. Trines, J. Vieira, A. J. W. Reitsma, R. Bingham, J. L. Collier, E. J. Divall, P. S. Foster, C. J. Hooker, A. J. Langley et al., Phys. Plasmas 13, 033108 (2006).

[31] R. M. G. M. Trines, C. D. Murphy, K. L. Lancaster, O. Chekhlov, P. A. Norreys, R. Bingham, J. T. Mendonça, L. O. Silva, S. P. D. Mangles, C. Kamperidis et al., Plasma Phys. Controlled Fusion 51, 024008 (2009).

[32] J. T. Mendonça, Theory of Photon Acceleration (CRC Press, Bristol, 2001).

[33] M. F. Kasim, N. Ratan, L. Ceurvorst, J. Sadler, P. N. Burrows, R. Trines, J. Holloway, M. Wing, R. Bingham, and P. Norreys, Phys. Rev. ST Accel. Beams 18, 032801 (2015).

[34] C. J. Dasch, Appl. Opt. 31, 1146 (1992).

[35] M. E. Taylor, Applied mathematical sciences 116, 1 (1996).

[36] N.H. Abel, Rolution de quelque problèmes à l'aide d'intégrales définies, 1823, Oeuvres Complètes, Vol. 1 (Christiania, Oslo, 1881), p. 11. 
[37] N. H. Abel, Résolution d'un problème de mecanique, 1826, Oeuvres Complètes, Vol. 1 (Christiania, Oslo, 1881), p. 97.

[38] N. H. Abel, Journal für die reine und angewandte Mathematik, 1, 153 (1826).

[39] A. M. Cormack, J. Appl. Phys. 34, 2722 (1963).

[40] R. A. Fonseca, L. O. Silva, F. S. Tsung, V. K. Decyk, W. Lu, C. Ren, W. B. Mori, S. Deng, S. Lee, T. Katsouleas et al., Lecture Notes in Computer Science Vol. 2329, III-342 (Springer, Heidelberg, 2002).

[41] R. G. Hemker, arXiv:1503.00276.
[42] C. K. Birdsall and A. B. Langdon, Plasma Physics via Computer Simulation (CRC Press, Bristol, 2004).

[43] C. W. Siders, S. P. Le Blanc, A. Babine, A. Stepanov, A. Sergeev, T. Tajima, and M. C. Downer, IEEE Trans. Plasma Sci. 24, 301 (1996).

[44] S. P. Le Blanc, E. W. Gaul, N. H. Matlis, A. Rundquist, and M. C. Downer, Opt. Lett. 25, 764 (2000).

[45] C. Iaconis and I. A. Walmsley, Opt. Lett. 23, 792 (1998).

[46] J. W. Goodman, Introduction to Fourier Optics (McGraw-Hill Book Company, New York, 1968). 